\title{
Will You Have a Good Sleep Tonight? Sleep Quality Prediction with Mobile Phone
}

\author{
Yin Bai ${ }^{* \dagger}$, Bin Xu *广, Yuanchao Ma*;, Guodong Sun $\ddagger$ Yu Zhao* \\ \{baiy,xubin,myc\}@keg.cs.tsinghua.edu.cn \\ gdsun.thu@gmail.com \\ zhaoyu121407@gmail.com
}

\begin{abstract}
Understanding the relationship between sleep and daily life can provide insights into a healthy life style since the sleep quality is one of the most important indicators of people's health status. This paper studies the extent to which a person's sleep quality can be predicted by his/her daily context information. A combination of the machine learning technology and medical knowledge is used to study the relation between context and sleep quality, so that sleep quality can be predicted in real time according to the relation.

We propose a novel sleep quality predicting framework from user context data, without requiring users to wear special devices. We develop a data collecting and analyzing prototype system called SleepMiner, which uses on-phone data such as mobile sensor data and communication data to extract human contexts. Then the relationship between context data and sleep quality is analyzed and a learning model based on factor graph model is proposed to predict sleep quality. From experimental results we demonstrate that it is possible to accurately infer sleep quality (around 78\%) from user context information. A set of solutions are proposed to address the practical problems of Android phone in data collection, making SleepMiner work with minimal impact on the phone's resources. We finally carry out experiments to evaluate our design in effectiveness and efficiency.
\end{abstract}

\section{Categories and Subject Descriptors}

H.4 [Information Systems Applications]: Miscellaneous

\section{General Terms}

Design

*Department of Computer Science and Technology, Tsinghua University, Beijing, China

${ }^{\dagger}$ Tsinghua National Laboratory for Information Science and Technology, Tsinghua University, Beijing 100084, China

${ }^{\ddagger}$ Information School, Beijing Forestry University, Beijing, China

Permission to make digital or hard copies of all or part of this work for personal or classroom use is granted without fee provided that copies are not made or distributed for profit or commercial advantage and that copies bear this notice and the full citation on the first page. To copy otherwise, to republish, to post on servers or to redistribute to lists, requires prior specific permission and/or a fee.

BodyNets' 2012 Oslo, Norway

Copyright 2012 ACM X-XXXXX-XX-X/XX/XX ...\$15.00.

\section{Keywords}

mobile phone, sleep quality prediction, factor graph model

\section{INTRODUCTION}

Sleep, as an important health indicator, connects tightly with some diseases $[9,5]$. Sleep is the best medicine, the traditional Chinese medicine praises and advocates highly. Most of existing works $[12,7]$ related to sleep quality are concerned about how to effectively measure sleep quality in clinic. And, a few works $[19,16]$ demonstrate the relationship between people's sleep quality and their contexts. In this paper, we focus on a more interesting and meaningful question: can we predict our sleep qualities using our daily context data (including both the people's physiological data and their surrounding profiles), or, informally, can we easily know in advance whether we will have a good sleep on the coming night? If we can mine the effects of context data on sleep quality without requiring people wearing external devices, the findings will be of great value for the judgment of health condition. Using the prediction result, we can provide useful health related services such as health status monitoring, health information recommending, etc. The method can also be extended to many other healthcare related applications.

Sleep quality can be divided into two types according to the duration of the measurements lasts; one is the longterm sleep quality, say, a month or more, and the other is at per-night level. Both sorts of sleep qualities are different in many respects, especially in the measurement methodology. In literature, the sleep quality refers to the former generally and is often assessed by self-rated questionnaires. For instance, the Pittsburgh Sleep Quality Index(PSQI) is a most widely used questionnaire and scoring tool for sleep quality evaluation [3]. In contrast, our study focuses on the per-night sleep quality prediction with only daily contexts automatically sampled from mobile phone. We argue that the prediction of per-night level is finer in time granularity and low in cost, thereby provisioning more real-time and efficient feedbacks to users or healthcare services.

In this paper, we propose a novel framework for sleep quality prediction based on mobile phone data. As we know, modern mobile phones are often fitted with various built-in sensors, like, GPS, three-axis accelerometers, photonic sensor, microphone, and the camera. Moreover, the communication logs, either from callings or short messages, can serve as a kind of soft sensor. These sensors are easy to profile the daily contexts of the carries, including their trajectory, surrounding noise, place, and even their social activities, social 
connections, and surroundings. Based on such features, we then investigate the effect of daily context information on the sleep quality and propose a factor graph model to predict the sleep quality. Finally we build a prototype (called SleepMiner) with Android platform to evaluate our work.

For testing the experimental result, a substantial sleep quality data as ground truth is needed. In our work a questionnaire assessing sleep quality is designed by us on the basis of PSQI, and a sleep quality detecting software is used as a complement to the questionnaire. The result of our experiments is then compared with the test data to verify the effectiveness of our method. Experimental results show that the sleep quality is inferred possibly from user context information with a fairly good accuracy (around 78\%). In summary, the contributions of our work are as follows:

1. The idea of predicting sleep quality with daily contexts releases users from wearing various medical devices, thereby making the users more comfortable and reducing the cost.

2. We design a factor graph model and use the user context features, which we extract from mobile phone data, to predict per-night sleep quality with a desirable accuracy (about $78 \%$ ).

3. We implement Android-based SleepMiner to evaluate our design. Especially we do a set of improvements for SleepMiner, which make it more friendly, energy-efficient, and robust. Specifically, we use a questionnaire based on PSQI and a sleep monitoring device to get the ground truth data for sleep quality evaluation.

The rest of the paper is organized as follows. Section 2 reviews the related work. Section 3 introduces the definitions of problem and the data. Section 4 talks about the data observation. Section 5 describes the proposed model and algorithm, in detail. Section 6 presents the SleepMiner system. Section 7 shows experiment results.Finally, Section 8 concludes this paper and talks about some future work.

\section{RELATED WORK}

In this section, we review related work on sleep quality. Existing works related with sleep quality are mainly about sleep quality detection with special device during sleep. There are also a number of works which analyze relationship between sleep health and factors such as certain diseases, physical and mental health, etc.

In [12], the authors develop a non-invasive, wearable neckcuff system capable of real-time monitoring and visualization of physiological signals. With this system one be able to monitor people's sleep continuously in a non-invasive and low cost method. The authors show one of the application$\mathrm{s}$ of their system which detects sleep apnea. [7] presents a sleep monitoring system based on the WISP platform-active RFID-based sensors equipped with accelerometers. The system accurately infers fine-grained body positions from accelerometer data collected from the WISPs attached to the bed mattress. Movements and their duration are also detected by the system.

Actigraphy [10] is a commonly used technique of monitoring human rest/activity cycles. It is used to clinically evaluate insomnia, circadian rhythm sleep disorders, excessive sleepiness and restless legs syndrome. During sleep, body movements will be monitored by the system [18] which is specially customized for the users. From the data collected by observing the movements of their wrist, sleep-wake states can be identified. Actiwatch is a wrist watch like device which measures limb movements using motion accelerometers. It has been used for monitoring motion-related sleep disorders $[14,13]$. In [2], the authors present a very accurate classifier of apnea episodes based on $\mathrm{SpO}_{2}$ signal that offers an accuracy of greater than $90 \%$. The authors also have implemented a system which is able to acquire the $\mathrm{SpO}_{2}$ signal, pre-process it, and identify the presence of sleep apnea in real time.

The main differences between existing work on sleep quality and our work are about three aspects. First, all these works focus on monitoring sleep quality during sleep but not predicting it. In our work we try to predict sleep quality using user context data such as daily activity, living environment and social activity information. Most of existing works aim at special diseases such as insomnia, circadian rhythm sleep disorders(CRSD) by monitoring sleep quality. We focus on how the user context information effects sleep quality. Moreover, most of previous works need special devices such as breast-band, wrist strap to monitor sleep quality, while in our work we use phone as the only client, without requiring participants to wear any other device.

There are also a number of works which analyze relationship between sleep health and both physical and mental health. [19] examines the sleep-health and lifestyle of elderly people in the prefecture of Okinawa. In [16] the factors that influence sleep health in junior high school students' lifestyles are examined. This piece of work is different from our work in that, they just illustrate that there exists some relationship between physical activities and sleep quality, no prediction is done using the data. They mainly focus on lifestyles such as physical exercises and special devices are also needed to detect the physical activity. In our work, we focus on predicting sleep quality by taking many kinds of context information into consideration. But, these works present a theoretical basis for our work.

\section{PRELIMINARY KNOWLEDGE}

As mentioned in section 1, PSQI is a widely used scoring tool of sleep quality but cannot be used in our work directly because it is designed for a long term sleep quality. We design the questionnaire of per-night sleep quality shown in the Table 1 on the basis of PSQI. PSQI contains nineteen selfrated questions which form seven component scores. Each component score has a range of $0-3$. The sum of the subscale scores yields a global score of sleep disturbance within 0 and 21. In our questionnaire, questions look like PSQI but most of them are closely concerned about the conditions of last day. It contains sixteen self-rated questions. We calculate the score of sleep quality using the Table 2, in which the notation "\#" means the sequence of question in Table 1. As shown, Table 2 consists of seven components, most of which have a score ranging from 0 to 3 , except questions 7 (whose score is either 0 or 1 ) and 8 (whose score is within 0 to 2). The summation of all components' scores calculated by Table 2 yields the final score of sleep quality that ranges from 0 to 18

Once we finish filling the Table 1 , we can calculate the final sleep quality score, according to the scoring scheme shown in Table 2. And, it is clear that the higher the final score is, the poor the sleep quality will be, and vice versa. Normally, the final score of the questionnaire are very meaningful in terms of health condition. "0-3" indicates a good sleep quality and thereby, you possess a good health with 
Table 1: Sleep Quality Questionnaire

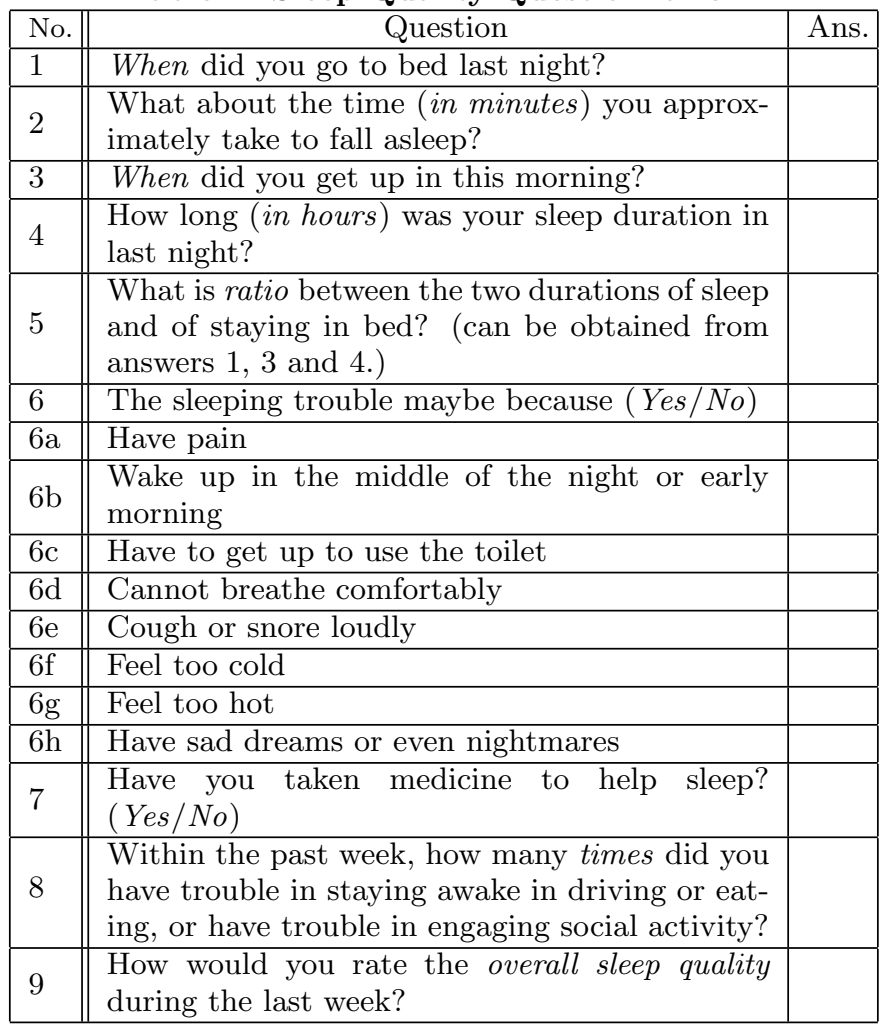

high probability. " $4-8$ " represents that your sleep quality is average and that if you don't pay enough attention, there will be a swift decline in your sleep quality. "9-16" shows that you possibly have sleep problems, and you must keep attention on such a situation. If the final score is greater than 16, your sleep quality is sure to be quite poor, and you are very likely to fall ill in near future.

Besides the questionnaire, we also take advantage of sleep quality monitoring software called "Sleep as Android" to collect subjects' sleep data. Similar to the "Sleep Cycle" [7] for iPhone, "Sleep as Android" is a popular application for Google Play Store. It monitors the subject during sleep and generates an objective sleep quality data. In our experiment, we require participants to open the Sleep-as-Android when they are going to sleep at night, and to put the phone on the bed. Specifically we use this application of sleep to complement the sleep quality scores calculated by our questionnaire.

\subsection{Problem Description}

The proposed sleep quality prediction scheme is based on context data collected by SleepMiner. First we describe subjects and the social activity between them using a graph. A social network can be represented as $G=(V, E)$, where $V$ is the set of $|V|=N$ subjects and $E \subset V \times V$ is the set of $|E|=M$ social activities between those subjects. We will define a set of attribute features extracted from mobile sensor data. All attribute features of subject $i$ in day $t$ is denoted as $X_{i}^{t}$. Specifically, $x_{i j}^{t}$ is the value of feature $j$ of subject $i$ in day $t$. Now we give the definitions of the attribute features in use. (1) Daily Trajectory. Daily trajectory means the track of one person in the whole day with
Table 2: Scoring Scheme for Some Questions

\begin{tabular}{|c|c|}
\hline \#2 & $\begin{array}{l}<\text { 16min: } 0,16-30 \mathrm{~min}: 1,31-60 \mathrm{~min}: 2 \text {, or }> \\
\text { 60min: } 3\end{array}$ \\
\hline \#3 & $>$ 7am: 0,6-7am: 1, 5-6am: 2 , or $<5 \mathrm{am:} 3$ \\
\hline$\# 5$ & $\begin{array}{l}>85 \%: 0,75 \% \sim 84 \%: 1,65 \% \sim 74 \%: 2, \text { or } \\
<65 \%: 3\end{array}$ \\
\hline \#6 & $\begin{array}{l}s=\text { sum of scores from } \# 6 \mathrm{a}, \# 6 \mathrm{~b}, \ldots, \text { up to } \# 6 \mathrm{~h} \\
\text { where } Y e s \text { and } N o \text { yield } 0 \text { and } 1 \text {, respectively. If } \\
s, 0: 0,1 \text { or } 2: 1,3 \sim 5: 2, \text { or } \geq 6: 3\end{array}$ \\
\hline \#7 & Yes: 1 and $N o: 0$ \\
\hline$\# 8$ & nill: 0, once or twice: 1 , or more than twice: 2 \\
\hline \#9 & $\begin{array}{l}\text { very good: } 0 \text {, fairly good: } 1 \text {, fairly bad: } 2 \text {, or very } \\
\text { bad: } 3\end{array}$ \\
\hline
\end{tabular}

corresponding timestamps. We get the trajectory from the GPS locations collected by SleepMiner. (2) Living Environment. In this study living environment mainly includes the sound and light information about the user's surroundings. Both of them are acquired from on-phone sensors. For each of them, we calculate the average over one day as its feature. (3) Activity. It means the postures of subject and in this paper it mainly covers walking, running, sitting, and standing. We sample accelerometer built in phone with a configured period of time, in order to extract subject's activity [1].

We specify the relationship between two subjects as features of social relevance, by using the mobile phone communication logs, if both subjects have connections. The relationship features of any two subjects are measured by the calling and message records between them, and is denoted by $E_{i j}^{t}=\left(e_{1}, e_{2}\right)$ where $e_{1}$ and $e_{2}$ are social features for calling and messaging, respectively. Similar to the sensorbased attributes feature, $e_{k} \in E_{i j}^{t}(k=1,2)$ measures the social activity feature $k$ for subjects $i$ and $j$ in day $t$. Besides these two above features, we also use the previous day's sleep quality of subject as the third feature which is denoted by $Y_{i}^{(t-1)}$ to represent the sleep quality of subject $i$ at the day $t-1$. In summary, status of a set of subjects can be modeled as a directed graph $G^{t}=\left\{V, E^{t}, X^{t}, Y^{(t-1)}\right\}$, where $V=\left\{v_{1}, v_{2}, \ldots, v_{n}\right\}$ is the set of subjects. $E \subseteq V \times V$ is the set of directed links between subjects. Each directed link $E_{i j}^{t} \in E$ is the social activity vector (social features) between subjects $v_{i}$ and $v_{j}$ for day $t$. Therefore the pernight sleep quality prediction problem in our study can be transformed into the following learning problem.

Sleep quality prediction. Let $\langle 1, \ldots, t>$ be a sequence of time stamps with the time granularity of day. Given the attribute feature set $X^{t}$, the previous night sleep quality $Y^{(t-1)}$, and the social feature $E^{t}$, the task is to find a predictive function

$$
f: G^{t} \rightarrow Y^{t}
$$

such that the sleep quality at time $t$ can be inferred.

Next we will present some significant observations that demonstrate the effectiveness of our features. And Section 5 will consider the combination of these features and then establish the learning model for predicting sleep quality.

\section{OBSERVATIONS}

In this section we will present some significant observations over a real-world dataset captured by SleepMiner. Figure 1 illustrates the sleep quality distribution of subjects. 


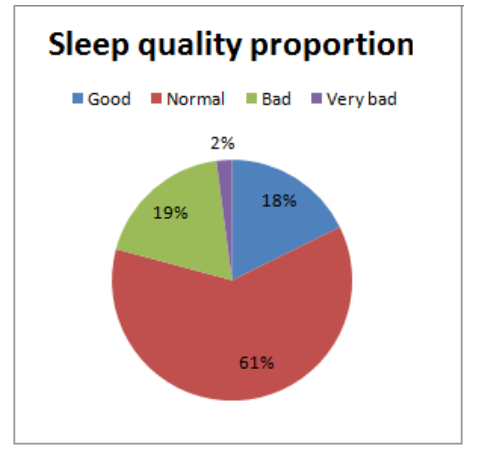

Figure 1: Sleep quality correlation

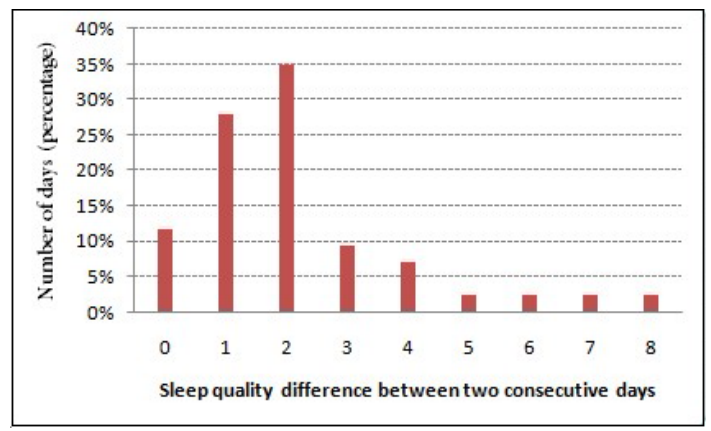

Figure 2: Sleep correlation distribution

We find that our participants have a normal sleep quality in most cases. The proportions of subjects with the good and bad sleep qualities are almost similar, both of which are less than $20 \%$, and there are few of our participants have poor sleep qualities.

We also investigate the relationship between the sleep qualities of two consecutive nights. The result is shown in Figure 2. We calculate the absolute value of the difference between sleep qualities of yesterday and the day before yesterday. We find from this figure that two consecutive sleep qualities are related but not so strong. For the scores for two consecutive nights, the difference between both of them in most cases are generally less than or equal to two points. In other words, the sleep qualities in two successive nights for a subject experiences no significant variation.

The correlation between the sensor features and the sleep quality has also been examined. In Figure 3(a) we find that one's sleep quality trends to become poor when his/her sitting time as a share of whole day is high. Additionally Figure 3(b) shows that if one stays in an environment with high sound intensity in day time, his/her sleep quality will be impacted, but slightly. We have similar observations from other contexts, like, the light intensity etc.; but we do not present these results due to the page limit. In summary we find out through preliminary investigations that a variety of human contexts correlates with the sleep quality in some degree, which motivates us to predict the per-night sleep quality based on the mobile data of daily relevance.

\section{MODEL FRAMEWORK}

Based on the above observation, we design a factor graph model $[8,6]$ to deal with the sleep-quality prediction at night level. We first define the following factor functions, the term

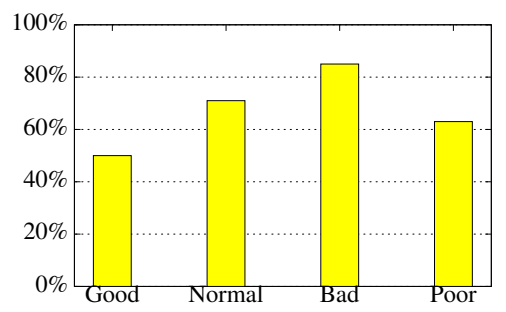

(a) Ratio of sitting posture

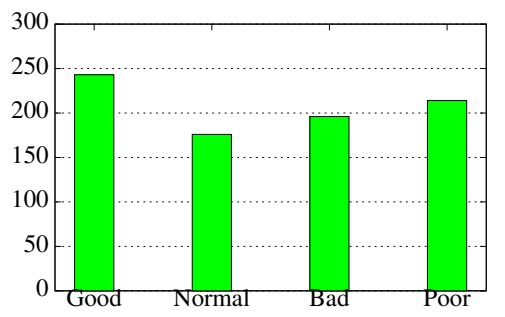

(b) Sound intensity

Figure 3: Correlation between the human activity as well as living environment and the sleep quality

of the factor graph theory, which weigh the effect of three sorts of features we consider.

(1) Attribute Factor: $f\left(y_{i}^{t}, \mathbf{x}_{i}^{t}\right)$ represents the posterior probability of the sleep quality $y_{i}^{t}$ given the the attribute vector $\mathbf{x}_{i}^{t}$. Specifically, $y_{i}^{t}$ represents the sleep quality of subject $v_{i}$ at time $t$, and $\mathbf{x}_{i}^{t}$ refers to the attributes of subject $v_{i}$ at time $t$. In particular, we define the attribute factor with

$$
f\left(y_{i}^{t}, \mathbf{x}_{i}^{t}\right)=\frac{1}{Z_{\alpha}} \exp \left\{\alpha^{T} \mathbf{f}\left(y_{i}^{t}, \mathbf{x}_{i}^{t}\right)\right\}
$$

where $\alpha$ is a weighting vector and $\mathbf{f}$ is a vector of feature functions.

(2) Social Correlation Factor: $g\left(y_{i}^{t}, G\left(y_{i}^{t}\right)\right)$ denotes the correlation between the social relationships, where $G\left(y_{i}^{t}\right)$ is the set of correlated relationships to $y_{i}^{t}$. That is, $g\left(y_{i}^{t}, G\left(y_{i}^{t}\right)\right)$ denotes the correlation between nodes via the edge on the graph model, which represents the social activity between two subjects in our model; $G\left(y_{i}^{t}\right)$ is the set of nodes' labels having relations to $y_{i}^{t}$. In particular, the social correlation factor can be expressed with

$$
g\left(y_{i}^{t}, G\left(y_{i}^{t}\right)\right)=\frac{1}{Z_{\beta}} \exp \left\{\sum_{y_{j}^{t} \in G\left(y_{i}\right)} \beta^{T} \mathbf{g}\left(y_{i}^{t}, y_{j}^{t}\right)\right\}
$$

where $\beta$ is a weighting vector and $\mathbf{g}\left(y_{i}^{t}, y_{j}^{t}\right)$ is a function to specify whether there is a link from node $i$ to node $j$ in the model; $\mathbf{g}\left(y_{i}^{t}, y_{j}^{t}\right)$ equals one if there is an edge from node $i$ to node $j$; otherwise 0 . In short, this social factor function aims at indicating the relations between any two nodes within the model.

(3) Sleep Correlation Factor: $h\left(y_{i}^{t}, H\left(y_{i}^{t-1}\right)\right)$ reflects the sleep quality correlation between two consecutive nights.

$$
h\left(y_{i}^{t}, H\left(y_{i}^{t-1}\right)\right)=\frac{1}{Z_{\gamma}} \exp \left\{\sum_{y_{i}^{t-1} \in H\left(y_{i}^{t}\right)} \gamma^{T} \mathbf{h}\left(y_{i}^{t}, y_{i}^{t-1}\right)\right\}
$$

where $\gamma$ is a weighting vector and $H\left(y_{i}^{t-1}\right)$ is the set of correlated relationships to $y_{i}^{t-1}$.

Given a network $G^{t}=\left\{V, E^{t}, X^{t}, Y^{(t-1)}\right\}$, we can define 
the joint distribution over $Y$ by

$$
p(Y \mid G)=\prod_{i} f\left(y_{i}^{t} \mathbf{x}_{i}^{t}\right) g\left(y_{i}^{t}, G\left(y_{i}^{t}\right)\right) h\left(y_{i}^{t}, H\left(y_{i}^{t-1}\right)\right)
$$

Moreover, given a set of labeled nodes in the factor graph, learning the model is just to estimate an optimal parameter configuration $\theta=\left(\alpha^{T}, \beta^{T}, \gamma^{T}\right)^{T}$ to maximize the loglikelihood function of $p(Y)$. For clarity, all feature functions for a node $y_{i}^{t}$ are concatenated as

$$
\mathbf{s}\left(y_{i}^{t}\right)=\left(\mathbf{f}\left(y_{i}^{t}, \mathbf{x}_{i}^{t}\right)^{T}, \sum_{y_{j}} \mathbf{g}\left(y_{i}^{t}, y_{j}^{t}\right)^{T}, \sum_{y_{j}} \mathbf{h}\left(y_{i}^{t}, y_{i}^{t-1}\right)^{T}\right)^{T}
$$

Based on Equations (1)-(6), the joint distribution $p(Y \mid G)$ can then be written as

$$
p(Y \mid G)=\frac{1}{Z} \exp \left\{\theta^{T} \sum_{i} \mathbf{s}\left(y_{i}^{t}\right)\right\}=\frac{1}{Z} \exp \left\{\theta^{T} \mathbf{S}\right\}
$$

where $Z=Z_{\alpha} Z_{\beta} Z_{\gamma}$ is a normalization factor and $\mathbf{S}=$ $\sum_{i} \mathbf{s}\left(y_{i}^{t}\right)$. Thus, the log-likelihood objective function can be expressed with

$$
\begin{aligned}
O(\theta) & =\log p\left(Y^{L}\right)=\log \sum_{Y \mid Y^{L}} \frac{1}{Z} \exp \left\{\theta^{T} \mathbf{S}\right\} \\
& =\log \sum_{Y \mid Y^{L}} \exp \left\{\theta^{T} \mathbf{S}\right\}-\log \sum_{Y} \exp \left\{\theta^{T} \mathbf{S}\right\}
\end{aligned}
$$

where $Y^{L}$ denotes the known labels and $Y \mid Y^{L}$ is a labeling configuration of $Y$ inferred from $Y^{L}$. A gradient decent method is adopted to maximize the object function. We calculate the gradient for each parameter $\theta$ by

$$
\begin{aligned}
\frac{\partial O(\theta)}{\partial \theta} & =\frac{\partial\left(\log \sum_{Y Y^{L}} \exp \left\{\theta^{T} \mathbf{S}\right\}-\log \sum_{Y} \exp \left\{\theta^{T} \mathbf{S}\right\}\right)}{\partial \theta} \\
& =\frac{\sum_{Y \mid Y^{L}} \exp \theta^{T} \mathbf{S} \cdot \mathbf{S}}{\sum_{Y \mid Y^{L}} \exp \theta^{T} \mathbf{S}}-\frac{\sum_{Y} \exp \theta^{T} \mathbf{S} \cdot \mathbf{S}}{\sum_{Y} \exp \theta^{T} \mathbf{S}} \\
& =\mathbb{E}_{p \theta\left(Y_{\left.\mid Y^{L}\right)} \mathbf{S}-\mathbb{E}_{p \theta(Y)} \mathbf{S}\right.}
\end{aligned}
$$

where $\mathbb{E}_{p \theta\left(Y \mid Y^{L}\right)} \mathbf{S}$ and $\mathbb{E}_{p \theta(Y)} \mathbf{S}$ are two expectations of $\mathbf{S}$ with and without labels, respectively, both of which cannot be directly calculated. In this paper, the Loopy Belief Propagation (LBP) [11] is employed to approximate marginal probabilities $p\left(y_{i} \mid \theta\right)$ and $p\left(y_{i}, y_{j} \mid \theta\right)$. The model learning algorithm is summarized in Algorithm 1.

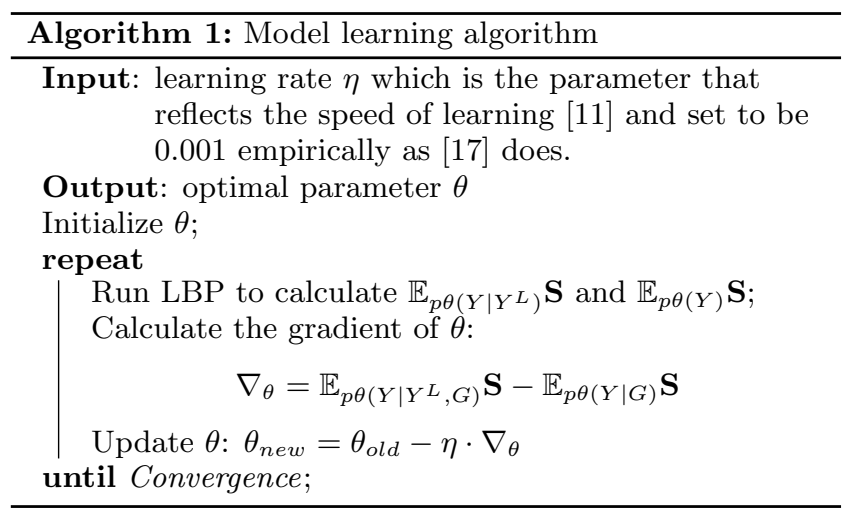

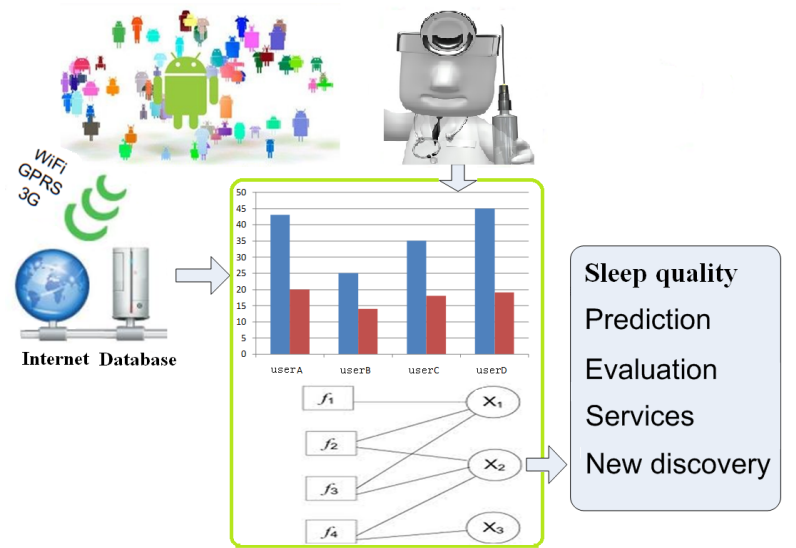

Figure 4: SleepMiner system architecture

Using the optimal parameter $\theta$ returned by Algorithm 1, we can infer the unknown labels by finding a label configuration that maximizes the joint probability $p(Y)$.

Finally, we use the largest marginal probability to predict the labels of nodes in our factor graph with the optimal $\theta$.

\section{SLEEPMINER IMPLEMENTATION}

For collecting and analyzing context data, we implement a mobile phone based system called SleepMiner, the architecture of which is shown in Figure 4. SleepMiner is compatible to any Android platform of version 2.2 or later.

\subsection{Android Platform Sensors}

Android is an open source Linux-based OS. It is now the most popular mobile phone system all over the world. In our experiments, we collect the raw data from the Android phone of version 4.0.4. The on-phone sensors are as follows. (1) Sound sensor. Android system does not provide an sound sensor. We get this type of data by turning to the microphone. For a series of sound sampling over a period of time, we can calculate the sum of raw samples (denoted by $s_{1}$ ) and the sum of the squared values of all the samples (denoted by $s_{2}$ ). With the total number of samples, say, $n$, we can calculate the strength of sound signal $S$ in dB by $S=\log _{10} \frac{s_{2}-s_{1}^{2} / n}{n}$. (2) Location. We acquire the longitude and latitude pairs by using the GPS module. In addition, the nearest WiFi device is also automatically detected by SleepMiner to assist in localizing. (3) Light sensor. The strength of light is returned by the light sensor equipped on almost all Android devices. In our experiment, SleepMiner detect$\mathrm{s}$ the light strength every 10 seconds, enough to reflect the variation of environmental light. (4) Accelerometer. Like GPS, accelerometer is also a very common sensing module for Android system. Besides collecting data, we use accelerometer to drive other sensors adaptively work. Specifically, we decide when to start or close the other sensors according to accelerations. The optimization policies will be detailed in section 6.3.

We use SleepMiner to collect subjects' context information, the way [15] dose, but mobile phone is the only client's device for SleepMiner, which collects daily life related data, such as sound, light, postures, and positions. Besides the on-phone sensory data, the communication logs covering the text and call records are also traced by SleepMiner without extracting the contents. 


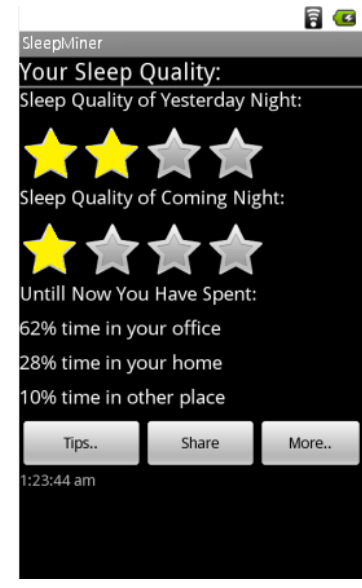

Figure 5: SleepMiner Application

\subsection{Software Components}

SleepMiner consists of two major parts: the client and the server. SleepMiner client is an Android application running on subjects' phone for collecting contexts. It generates the data and sends it to SleepMiner server which stores the data in structured form and deals with data analysis. The default data acquisition frequency is once every 10 seconds. Subjects in our experiment should run the SleepMiner clien$\mathrm{t}$ application on their phone in a whole day except sleeping time, and fill out the questionnaire every morning about the sleep quality of last night. To encourage the subjects to use our application, we feedback some information to them about their sleep quality and some other related information. Figure 5 shows the user interface of our application.

SleepMiner server takes the charge of data storage and analysis. To improve the efficiency of our system, we implement the following functions on our system. (1) The system provides a analysis report to all subjects every day, which includes some basic statistical information such as data size. For those who didn't collect data successfully due to some personal reasons, the system will remind them by feed-backing to these subject a notification. (2) Sometimes, there exists some obviously abnormal data in the raw data. The system pre-processes the data set every day and removes the abnormal data. (3) SleepMiner server provisions friendly user interfaces to visualize the data and to fetch the data; these interface make the data analyzing very easy.

\subsection{Implementation Considerations}

To save energy we take advantage of methods proposed in [4]. We try to reduce the running time of GPS sensor, which consumes a lot of electricity. The system detects that the subject is standing or sitting for a while, and then it close the sensor until the system detect that subject is running or walking again. This strategy is of great use because people always do not move much during office time.

To improve the network communication quality, we design and implement following strategies: (1) In client, SleepMiner generates data in JSON format, which enables the sending of the data relatively quick and compact. With JSON, it is also easy to parse the data on the server since there are many existing tool to parse on it. (2) We transmit data periodically rather than in strict real-time. SleepMiner client stores the data when generating it and sends the data to server once every hour. SleepMiner client also backups the data in client phone's SD card to prevent possible errors and data loss. Using this strategy also satisfies the requirement of real time prediction of sleep quality in coarse grain and more importantly, reduces the energy cost in network communication significantly. (3) For reducing the communication time, SleepMiner uses the socket communication rather than the HTTP POST sending mode. The SleepMiner server is also designed to response the client request immediately after receiving data, then to parse as well as document the data. In comparison with the general solution in which the server responses the client request after parsing the data, our implementation reduces response time obviously and consequently improves the reliability of network connection.

\section{EXPERIMENTS}

Our experiments are carried out with 15 subjects for 30 successive days, sampling phone-based context data covering physiological activities, surroundings, and social activities. During the daytime, all subjects are required to switch on the SleepMiner client installed on their phone. Every morning, they are also needed to fill the questionnaire of sleep quality relevance. Especially, we validate each questionnaire submitted every day in terms of conflicts, in order to ensure that subjects carefully finish the questionnaire. Validation results show that more than $98 \%$ of the questionnaires are filled out carefully by subjects. In addition, we require that subjects run the Sleep-As-Android application when they are ready to go to bed.

The overall experimental results (in Table 3) show that the performance of our model can achieve around $78 \%$ in terms of the quality of sleep quality prediction.

Table 3: Performance of SleepMiner assessment.(\%)

\begin{tabular}{|l||c|c|c|r|}
\hline Factors used & Accuracy & Precision & Recall & F1-score \\
\hline \hline Previous day & 59.42 & 67.50 & 79.41 & 72.97 \\
\hline + Attributes & 73.91 & 84.85 & 82.35 & 83.58 \\
\hline + Social & 65.22 & 71.05 & 79.41 & 75.00 \\
\hline All & 78.26 & 87.50 & 82.35 & 84.85 \\
\hline
\end{tabular}

We evaluate the contribution of different factors defined in our model as follows. We first remove attribute correlation factors and social correlation factors and only keep the sleep correlation factor, and then add each of the factors into the model. Then we evaluate the performance improvement by each factor. Table 3 shows that we achieve a relative high accuracy when we take all the features into consideration. We find that all the factors are useful for predicting the sleep quality, but the contribution is difference. Specifically, with the sleep correlation factor only, the accuracy would achieve to almost $60 \%$. After adding the attribute factor, we get a significant improvement of accuracy, of about $14 \%$, which means the peoples' daily activity and living environments have a obvious effects to the subjects' sleep quality. This, also proved the effectiveness of our work. From the result we also find that social factor affects the accuracy about $6 \%$, but not as much as attribute factor. It's partially because we describe the social activity in our model only using the calling record and SMS record, while a person's social activity can include much more things. By combining all the factors together, we can further obtain a accuracy of $78 \%$, that's because our model not only considers different factors, but also leverages the correlation between them. 
Note that in fact, there exist differences in some respects over subjects which will bring uncertainties in some degree. For example, subjects have different habits of using their phone, different surroundings, and different reaction to surroundings; so we will in future improve our design by adapting the model parameters to subject. Additionally, there are a few other factors possibly affecting the accuracy of sleepquality prediction, such as the irregular, sporadic sleep time of subject.

\section{CONCLUSIONS}

In this paper, we propose an novel approach for predicting individual's sleep quality using mobile data. We first define the problem by designing a questionnaire based on PSQI. We extract several features such as daily activity, living environment and social activity from mobile phone data, and then propose a method based on factor graph for predicting sleep quality using these features. For evaluating the proposed model, the SleepMiner system is developed, which uses mobile as the only client and collects context data in real time. The system implements several strategies to improve the efficiency of data collecting and reliability of network communication. We train our model using context features, the experimental result shows that the proposed factor graph model can significantly improve the performance for sleep quality prediction.

In future, we will take other factors into consideration such as people for different groups, etc.. For different people, the effects of daily contexts on their sleep quality might be different. So we will, in the future, focus on a few special groups of people such as the elder and ones with a sort of diseases. In addition, because the accuracy of mobile data is not fully accessed all the time, we will try to employ some other commercial-off-the-shelf devices if they can enable an improvement in prediction quality.

\section{ACKNOWLEDGMENTS}

This work is supported in part by the NSF of China granted 61170212 and by the China Post-doctor Science Foundation (Special Program) granted 201104101.

\section{REFERENCES}

[1] L. Bao and S. Intille. Activity recognition from user-annotated acceleration data. In LNCS 3001, pages 1-17, 2004.

[2] A. Burgos, A. Goñi, A. Illarramendi, and J. Bermúdez. Real-time detection of apneas on a pda. Trans. Info. Tech. Biomed., 14(4):995-1002, July 2010.

[3] D. J. Buysse, C. F. Reynolds, T. H. Monk, S. R. Berman, and D. J. Kupfer. The pittsburgh sleep quality index: a new instrument for psychiatric practice and research. Psychiatry Research, 28(2):193-213, 1989.

[4] J. Cui, G. Sun, and B. Xu. Ad-sense: activity-driven sensing for mobile devices. In SenSys, pages 399-400, 2011.

[5] S. A. Factor, T. McAlarney, J. R. Sanchez-Ramos, and W. J. Weiner. Sleep disorders and sleep effect in parkinson's disease. Movement Disorders, 5:280-285, 1990.

[6] J. Hopcroft, T. Lou, and J. Tang. Who will follow you back? reciprocal relationship prediction. In
Proceedings of the 20th ACM international conference on Information and knowledge management, CIKM '11, pages 1137-1146, New York, NY, USA, 2011. ACM.

[7] E. Hoque, R. F. Dickerson, and J. A. Stankovic. Monitoring body positions and movements during sleep using wisps. In Wireless Health 2010, WH '10, pages 44-53, New York, NY, USA, 2010. ACM.

[8] F. R. Kschischang, B. J. Frey, and H.-A. Loeliger. Factor graphs and the sum-product algorithm. IEEE Transaction on Information Theory, 47:498-519, Feb. 2001.

[9] R. S. T. LEUNG and T. D. BRADLEY. Sleep apnea and cardiovascular disease. American Journal of Respiratory and Critical Care Medicine, 164(12):2147-2165, 2001.

[10] T. Morgenthaler, C. Alessi, L. Friedman, J. Owens, V. Kapur, B. Boehlecke, T. Brown, A. Chesson, J. Coleman, T. Lee-Chiong, and et al. Practice parameters for the use of actigraphy in the assessment of sleep and sleep disorders: an update for 2007. Sleep (Rochester), 30(4):519-529, 2007.

[11] K. P. Murphy, Y. Weiss, and M. I. Jordan. Loopy belief propagation for approximate inference: An empirical study. In UAI, pages 467-475, 1999.

[12] M. Rofouei, M. Sinclair, R. Bittner, T. Blank, N. Saw, G. DeJean, and J. Heffron. A non-invasive wearable neck-cuff system for real-time sleep monitoring. In Proceedings of the 2011 International Conference on Body Sensor Networks, BSN '11, pages 156-161, Washington, DC, USA, 2011. IEEE Computer Society.

[13] A. Sadeh, K. M. Sharkey, and M. A. Carskadon. Activity-based sleepĺcwake identification: An empirical test of methodological issues. Sleep, 17:201-207, Apr. 1994.

[14] T. Salmi and L. Leinonen. Automatic analysis of sleep records with static charge sensitive bed. Electroencephalogr. Clin. Neurophysiol., 64:84-87, July 1986.

[15] C. Seeger, A. Buchmann, and K. V. Laerhoven. myhealthassistant: A phone-based body sensor network that captures the wearer's exercises throughout the day. In BodyNets, 2011.

[16] H. Tanaka, K. Taira, M. Arakawa, A. Masuda, Y. Yamamoto, Y. Komoda, H. Kadegaru, and S. Shirakawa. An examination of sleep health, lifestyle and mental health in junior high school students. Psychiatry and Clinical Neurosciences, 56:235-236, June 2002.

[17] W. Tang, H. Zhuang, and J. Tang. Learning to infer social ties in large networks. In D. Gunopulos, T. Hofmann, D. Malerba, and M. Vazirgiannis, editors, ECML/PKDD (3), volume 6913 of Lecture Notes in Computer Science, pages 381-397. Springer, 2011.

[18] W. W. Tryon. Issues of validity in actigraphic sleep assessment. Sleep, 27:158-165, Feb. 2004.

[19] E. Uezu, K. Taira, H. Tanaka, M. Arakawa, C. Urasakii, H. Toguchi, Y. Yamamoto, E. Hamakawa, and S. Shirakawa. Survey of sleep-health and lifestyle of the elderly in okinawa. Psychiatry and Clinical Neurosciences, 54:311-313, June 2000. 